\title{
Development of iron (Fe) additions approach for cost reduction in Ti-alloy - a review
}

\begin{abstract}
Reduction of materials cost without sacrificing their functional properties is one of the main goals for materials engineers. This article focuses on Fe and its effect as an obvious example of introducing inexpensive alloying elements into Ti-alloys. It could also be a guide for future researches that would be aimed at replacing expensive $\beta$-stabiliser elements with inexpensive elements. Recently, more attention has been paid to low-cost Ti-alloys that contain Fe. This has resulted in the designing of many alloys such as Ti metal $62 \mathrm{~S}$ and Ti-Fe-O-N Ti-alloys. This technical trend has been used to design new Ti-alloys with good mechanical features, such as Ti8LC and Ti-5.5Al-1Fe Ti-alloys in China and Japan respectively. Nowadays, major composition modifications of Ti-6Al-4V alloy have been proposed through the complete replacement of $\mathrm{V}$ by Fe. Three new alloys (Ti-6Al-xFe) are developed to investigate the effect of Fe additions on the microstructure and the mechanical properties.
\end{abstract}

Keyword: Fe addition; Ti-alloy; Cost reduction; -Stabilising alloying elements 